Exp. Anim. 55(5), 415-418, 2006

\title{
Comparison of Rat Mandible Bone Characteristics in F344 Substrains, F344/Du and F344/N
}

\author{
Shin TANAKA ${ }^{1)}$, Sachi KUWAHARA ${ }^{1,2)}$, Kazutoshi NISHIJIMA1), \\ Tamio $\mathrm{OHNO}^{3)}$, Masahiro NAGAYA ${ }^{4)}$, Yasunori NAKAMURA ${ }^{5)}$, Yasunori SUMI), \\ Osamu MIYAISHI ${ }^{6}$, Hiroaki AOYAMA ${ }^{7)}$, and Nobuo GOTO ${ }^{8)}$
}

\begin{abstract}
1)Animal Facility for Aging Research (AFAR), National Institute for Longevity Sciences (NILS), 474-8522, 4)Department of Rehabilitation, 5)Division of Oral and Dental Surgery, Department of Advanced Medicine, National Hospital for Geriatric Medicine, National Center for Geriatrics and Gerontology (NCGG), 36-3 Gengo Morioka-cho, Obu 474-8511, 2)Department of Anatomy, Hyogo College of Medicine, 1-1 Mukogawa-cho, Nishinomiya, 663-8501, 3)Division of Experimental Animals, Center for Promotion of Medical Research and Education, Graduate School of Medicine, Nagoya University, 65 Tsurumai-cho, Showa-ku, Nagoya 466-8550, 6) Chubu Rosai Hospital, 1-10-6 Koraku, Minato-ku, Nagoya 455-8530, 7)Institute for Environmental Toxicology, 4321 Uchimoriya-cho, Joso 303-0043, and ${ }^{8}$ Japanese Society for Laboratory Animal Resources 2-8-10
\end{abstract}

Iwamoto-cho, Chiyoda-Ku, Tokyo 101-0032, Japan

\begin{abstract}
The characteristics of the mandible bone were compared through DXA methods between two major substrains of F344 rats, F344/DuCrICrlj and F344/NSIC at around 60 days of age. Since these two substrains are clearly different in survival and mandible morphology, some genetic differences are supposed to exist. In contrast to a previous microsatellite analysis, clear and significant differences were detected in the body and mandible weights, the mandible bone mineral contents (BMC), bone area (AREA), bone mineral density (BMD) and bone mineral ratio (BMR), between F344/DuCrICrlj and F344/ NSIc, with the mandible molar teeth intact in the bone. Thus, care is needed in the experimental use of these substrains, as results may differ between them. The newly proposed parameter, BMR, may especially contribute to the comparison of bone characteristics among species.
\end{abstract}

Key words: Bone Mineral Density: BMD, Bone Mineral Ratio: BMR, mandible, substrain difference

\section{Introduction}

The longevity sciences, especially the geriatric medicine, need practical and contributive animal models. The most convenient animal models, laboratory rodents, however, sometimes bring unexpected confusion to experimental results due to their species, strain and sex specific biological characteristics, especially their aging properties. Survival, a reliable parameter for the establishment of the aged individuals and the monitor-

(Received 11 April 2006 / Accepted 19 June 2006)

Address corresponding: S. Tanaka, Animal Facility for Aging Research (AFAR), National Institute for Longevity Sciences (NILS), 36-3 Gengo, Morioka-cho, Obu, Aichi 474-8522, Japan 
ing of the aging process, was found to be different among F344 substrains and between the USA and Japan [3]. Namely, the survival of F344/N was different from that of F344/Du in Japan and the survival of F344/ $\mathrm{Du}$ was different between Japan and USA[3, 4]. Microsatellite analysis, however, demonstrated that these two substrains in the two countries were the same [4, unpublished observation]. Morphometric analysis of the mandible shape, known to be effective for the detection of strain differences, demonstrates clear substrain differences among F344 rats [submitted for publication to this journal]. The current research was conducted to compare the mandible bone with special attention to its characteristics including the bone mineral contents (BMC), bone area (AREA) and bone mineral density (BMD) between two F344 substrains available in Japan, F344/DuCrlCrlj and F344/NSlc. These parameters are fundamental to the evaluation of animal models and are related to bone metabolism. Interestingly, these two substrains are identifiable by the shape of their face among F344/NSlc, F344/NHsd and F344/DuCrlCrlj [Tanaka and Miyaishi, unpublished observation].

\section{Materials and Methods}

\section{Experimental animals}

All animals were male and used at 2 months of age. Two substrains of F344 rats, F344/Du ( $n=11$, Table 1) and $\mathrm{F} 344 / \mathrm{N}(\mathrm{n}=10$, Table 1), were purchased from Charles River Laboratories Japan (CRLJ), Yokohama, Japan and SLC Japan (SLC), Hamamatsu, Japan, respectively. Thus, the substrains of "F344" used were F344/DuCrlCrlj and F344/NSlc [1, 3, 4]. The former was fed CRF1 (Crude protein contents: 22.4\%, Oriental Yeast, Tokyo) and the latter was fed MRA2 (Current RA2) (Crude protein contents: 22.7\%, Nosan, Yokohama), at CRLJ and SLC until the supply, respectively. The substrains are subsequently described only as F344/Du and F344/N, respectively. Animals were housed in groups after weaning at each breeder.

All animal experiments were performed with the permission of the Committee for Animal Ethics NILS according to the Guideline of NILS Animal Experimentation with consideration to animal rights and welfare.

\section{Preparation of the mandible}

Animals were sacrificed by an overdose of anesthesia on either 58 or 59 days of age for F344/N, and 61 or 62 days of age for F344/Du on the delivery day after weighing, and the whole tissue around the oral cavity was dissected out. The dissected tissue was autoclaved at $121^{\circ} \mathrm{C}$ for $5 \mathrm{~min}$. The soft tissue around the mandible was removed carefully. The mandible was incubated overnight in $0.5 \%$ papain (Merck, Germany) solution at $37^{\circ} \mathrm{C}$ and the attaching soft tissue was digested. The incisors were pulled out. After washing and drying, a dried bone specimen of the mandible was prepared.

\section{Measurement of mandible}

The mandibles without the incisors were weighed. The whole mandible was measured and surveyed by the DXA method, with DCS-600EX-IIIR, ALOKA, Tokyo.

The bone mineral contents (BMC) in $\mathrm{mg}$, bone area (AREA) in $\mathrm{cm}^{2}$, and bone mineral density (BMD) in $\mathrm{mg} / \mathrm{cm}^{2}$ were measured. The ratio of the BMC to mandible weight was also calculated. These parameters were measured with the three mandible molar teeth intact in the mandible. The incisors were pulled out in the specimen preparation. The mandible weight and BMC, thus, contained both bone and tooth weight and calcium (hydroxy) apatite.

\section{Statistical procedures}

The data obtained were compared by Student's $t$-test after the $\mathrm{F}$ test.

\section{Results}

\section{Body and mandible weights}

As summarized in Table 1, the body weights of F344/ Du varied from 181.3 to $196.8 \mathrm{~g}$ and the average \pm SD was $190.3 \pm 4.9 \mathrm{~g}$. In F344/N, the body weights ranged from 167.7 to $181.7 \mathrm{~g}$ and the average was $175.7 \pm 4.9$ g. The body weight was significantly $(P<0.001)$ larger in F344/Du than in F344/N.

Similarly, the average mandible weight of F344/Du varied from 173.3 to $184.6 \mathrm{mg}$ and $179.8 \pm 2.9 \mathrm{mg}$, and in $\mathrm{F} 344 / \mathrm{N}$ it varied from 162.5 to $175.2 \mathrm{mg}$ and its average \pm SD was $170.3 \pm 4.8 \mathrm{mg}$. The mandible weight was significantly $(P<0.001)$ larger in F344/Du than in F344/N. 
Table 1. Weight, BMC, AREA, BMD and BMR of Mandible on two F344 Substrains, F344/Du and F344/N

\begin{tabular}{|c|c|c|c|c|c|c|c|}
\hline Substrain & Days of age & Body weight & Mandible weight & $\begin{array}{l}\text { Bone mineral } \\
\text { contents }\end{array}$ & Bone area & $\begin{array}{l}\text { Bone mineral } \\
\text { density }\end{array}$ & $\begin{array}{l}\text { Bone mineral } \\
\text { ratio }\end{array}$ \\
\hline & & g & $\mathrm{mg}$ & BMC: $\mathrm{mg}$ & AREA: $\mathrm{cm}^{2}$ & BMD: $\mathrm{mg} / \mathrm{cm}^{2}$ & BMR: ratio \\
\hline \multicolumn{8}{|l|}{ F344/DuCrlCrlj } \\
\hline $\begin{array}{l}\mathrm{n}=11 \\
\text { range }\end{array}$ & $61 / 62$ & $181.3-196.8$ & $173.3-186.4$ & $95.3-103.0$ & $1.509-1.611$ & $62.0-64.3$ & $0.546-0.558$ \\
\hline average \pm SD & & $190.3 \pm 4.9$ & $179.8 \pm 2.9$ & $99.1 \pm 2.1$ & $1.558 \pm 0.034$ & $63.6 \pm 0.7$ & $0.551 \pm 0.004$ \\
\hline \multicolumn{8}{|l|}{ F344/NSlc } \\
\hline $\begin{array}{l}\mathrm{n}=10 \\
\text { range }\end{array}$ & $58 / 59$ & $167.7-181.7$ & $162.5-175.2$ & $89.4-96.9$ & $1.470-1.550$ & $60.6-63.3$ & $0.542-0.553$ \\
\hline average $\pm \mathrm{SD}$ & & $175.7 \pm 4.9$ & $170.3 \pm 4.8$ & $93.2 \pm 2.8$ & $1.507 \pm 0.030$ & $61.9 \pm 1.1$ & $0.547 \pm 0.004$ \\
\hline$t$-test & & $P<0.001$ & $P<0.001$ & $P<0.001$ & $P<0.01$ & $P<0.001$ & $P<0.05$ \\
\hline
\end{tabular}

\section{Bone mineral contents (BMC)}

The bone mineral contents (BMC) of the F344/Du mandible varied from 95.3 to $103.0 \mathrm{mg}$ and were 99.1 $\pm 2.1 \mathrm{mg}$ on average. The BMC of the F344/ $\mathrm{N}$ mandible varied from 89.4 to $96.9 \mathrm{mg}$ and was $93.2 \pm 2.8$ $\mathrm{mg}$ on average. The $\mathrm{BMC}$ was significantly $(P<0.001)$ larger in F344/Du.

\section{Bone area (AREA)}

The area (AREA) of the mandible in F344/Du varied from 1.509 to $1.611 \mathrm{~cm}^{2}$ and was $1.558 \pm 0.034 \mathrm{~cm}^{2}$ on average. The AREA of the F344/N mandible ranged from 1.470 to 1.550 and was $1.507 \pm 0.030 \mathrm{~cm}^{2}$ on average. The AREA was significantly $(P<0.01)$ larger in F344/Du.

\section{Bone mineral density (BMD)}

The bone mineral density (BMD), which was calculated by dividing the BMC with AREA of the F344/Du mandible ranged from 62.0 to 64.3 and was $63.6 \pm 0.7$ $\mathrm{mg} / \mathrm{cm}^{2}$ on average. The BMD of the $\mathrm{F} 344 / \mathrm{N}$ mandible ranged from 60.6 to 63.3 and was $61.9 \pm 1.1 \mathrm{mg} /$ $\mathrm{cm}^{2}$ on average. The BMD was significantly $(P<0.001)$ larger in F344/Du.

\section{The ratio of $B M C$ to the mandible weight (BMR)}

The ratio of the BMC to the mandible weight, which was calculated by dividing the $\mathrm{BMC}$ with the mandible weight, was ranged from 0.546 to 0.558 and $0.551 \pm$ 0.004 on average for $\mathrm{F} 344 / \mathrm{Du}$; it ranged from 0.542 to 0.553 and $0.547 \pm 0.004$ on average for F344/N. This ratio was named $\mathrm{BMR}$, bone mineral ratio, and it was significantly $(P<0.05)$ larger in $\mathrm{F} 344 / \mathrm{Du}$.

\section{Discussion}

For all of the parameters, body and mandible weights, bone mineral contents (BMC), bone area (AREA), bone mineral density (BMD), and the ratio of BMC to mandible weight (BMR), measured or calculated in the present study, statistically significant differences were detected between the two substrains, F344/Du (F344/ DuCrlCrlj) and F344/N (F344/NSlc) of the inbred strain of rats, F344 (Table 1). These two substrains, F344/N and $\mathrm{F} 344 / \mathrm{Du}$, were segregated from the original line at Columbia University by transfer to the NIH (National Institutes of Health) in 1949 and to CRL (Charles River Laboratories) in 1976, respectively [1, 3]. In addition to the present results, the survival and disorders with aging are known to be clearly different $[2,3]$. The presence of some genetic differences is strongly suggested to be present between these two substrains. However, microsatellite analysis did not detect differences among the four substrains between Japan and USA [4, unpublished observation]. Thus, care is needed in the experimental use of these two substrains, as results may differ between them.

All parameters measured and calculated, especially the body and mandible weights and BMC, were significantly larger in F344/Du (Table 1). This may, in part, be due to the acute growth in F344/Du. The background of this biological characteristic including the 
aging should be investigated with special attention paid to the aging changes. It is generally accepted that the later maturity brings longer survival. However, F344/ Du survive longer than F344/N [3]. F344/N is characterized by a high incidence of mono nucleated lymphocyte leukemia [2]. A disorder with aging, that is also inherent, may seriously modify the survival or the aging process. The strain or substrain characteristics should be noted and recognized before the usage of these substrains of rats.

The mandibular bone properties observed in the present study seemed to be dependent on growth, except for BMR (Table 1). Some significant substrain differences seem to depend on growth, especially at the age selected for this experiment. An unexpected finding was that of the BMR. A significant substrain difference was also detected in this index, even though both the mandible weight and BMC were significantly different. This index, BMR, was devised to eliminate the substrain differences of these two parameters. However, the BMR showed a significant substrain difference. This means the ratio or the proportion of calcium apatite per unit of mandible bone weight is significantly different between the two substrains. This significant substrain difference in BMR may reflect a difference in bone metabolism, namely a genetic difference. Microsatellite analysis did not detect differences between the two substrains; however, various physiological differences, including the mandible bone properties strongly suggest the presence of a genetic difference. This also raises questions over the experimental usage of these substrains. Since the BMR was calculated for mandibles containing molar teeth, this index should be reassessed using different bones like the femur.

In the present study, a new index, BMR was devised (Table 1). The BMD was calculated by dividing the BMC by the AREA. Usually, the density is calculated by dividing the mass by the volume. The BMD thus calculated, varies according to the size of the bone. To eliminate this discrepancy, the BMC was divided by the bone weight in the present study. This index is expected to reflect the real and direct bone physiology.
The bone mineral ratio, BMR, will contribute to the comparison of bone properties among various animal species. The BMR will contribute to recalculate the bone weight from the group of the BMC, AREA and BMD. As a convenient index, BMR should make a great contribution both aging and osteoporosis researches.

Attention should be paid to the fact that the mandible BMC included the calcium apatite content of three molar teeth. Research is underway now to identify the $\mathrm{BMC}$ of the mandible bone proper.

In conclusion, major substrain differences were identified in the mandible bone characteristics of F344 rats. The results strongly suggests that the two substrains, F344/DuCrlCrlj and F344/NSlc, are different to each other not only physiologically but also genetically.

\section{Acknowledgments}

This report was supported in part by the Research Grant for Longevity Sciences (14C-5) from the Ministry of Health, Labour and Welfare. The authors wish to express their sincere thanks to Dr. Toshiki OHTA, President, National Hospital for Geriatric Medicine, for his kind advice, to Dr. Megumi SASAKI, Department of Gerontological Policy, for her advice in the statistics and to Mrs. Haruko SUZUKI for her help on the preparation of the specimens.

\section{References}

1. Festing, M.F.W. 1979. Genetic Quality Control. pp. 36-53. In: Inbred Strains in Biomedical Research (Festing, M.F.W ed.), The MacMillan Press, London and Baskingstoke.

2. Miyaishi, O., Tanaka, S., Kanawa, R., Matsuzawa, K., and Isobe, K. 2000. Anisocytosis precedes onset of the large granular lymphocyte leukemia in aged F344/N rats. Arch. Geront. Geriat. 30: 161-172.

3. Tanaka, S., Shito, A., Tamaya, N., Miyaishi, O., Nishimura, M., and Ohno, T. 2002. Difference in average survival between F344/Du and F344/N rats is not due to genetic contamination. Arch. Geront. Geriat. 34: 19-28.

4. Tanaka, S., Tamaya, N., Matsuzawa, K., and Miyaishi, O. 2000. Differences in survivability among F344 rats. Exp. Anim. 49: 141-145. 